\title{
Helical Computed Tomography in Evaluation of Selected Cases of Acute Abdomen
}

\author{
Saddig D. Jastaniah, Alamin M. Salih \\ Diagnostic Radiology Department, King Abdulaziz University, Jeddah, Saudia Arabia \\ Email: sjastaniah@kau.edu.sa
}

Received 17 August 2014; revised 15 September 2014; accepted 22 September 2014

Copyright (C) 2014 by authors and Scientific Research Publishing Inc.

This work is licensed under the Creative Commons Attribution International License (CC BY).

http://creativecommons.org/licenses/by/4.0/

(c) (i) Open Access

\begin{abstract}
Acute abdomen is a common presentation in emergency medicine. It represents $5 \%$ to $10 \%$ of all Emergency Department (ED) visits. Diagnosis by imaging includes digital X-ray unit, sonography (US) unit and computed tomography (CT) equipment. During the last years, a trend towards increased use of computed tomography in patients with acute abdomen can be seen. Additionally, patient with severe claustrophobic often cannot tolerate MR scanner. The aim of the present study was to investigate the possibility of optimizing Helical CT parameters in the protocol and emphasize the CT features of selected cases of disorders related acute abdominal complain at the Emergency Department both in general and in a number of selected conditions (Urolithiasis, Aortic Aneurysm Rupture and acute cholecystitis). According to this work findings, non-contrast CT after ultrasound is diagnostic modality for patients with urinary stones in the Emergency Department. Contrast-enhanced CT was highly sensitive for acute aortic syndrome and therefore the CT imaging protocols must be adjusted in order to minimize dose from radiation.
\end{abstract}

\section{Keywords}

Acute Abdomen, CT, Acute Aortic Syndrome, Urinary Calculi

\section{Introduction}

The clinical syndrome of acute abdomen is characterized by a sudden severe abdominal pain that sometimes evolve emergency treatment or surgical interference. A definitive method of diagnosis is a must to obtain cure [1]. The differential diagnosis has a wide range of disorders varying from benign diseases to cases requiring 
immediate surgery [2].

Acute aortic syndromes can be aortic dissection, ruptured aortic aneurysm, penetrating atherosclerotic ulcer, and intramural hematoma. These illnesses present similarly and have incidence estimates of two to four cases per 100,000 people per year [3] [4]. Rapid and accurate diagnosis is essential to improve survival because acute aortic dissection has a pre- and in-hospital mortality rate of $20 \%$ and $30 \%$, respectively [4].

Several imaging modalities can be used, including MRI and transesophageal echocardiography, but CT has emerged as the first choice given its availability, speed, and accuracy with sensitivity and specificity approaching $100 \%$ in diagnosis of acute aortic syndromes (aortic dissection, intramural hematoma, penetrating atherosclerotic ulcer, and ruptured aortic aneurysm) [5].

Helical scanning CT has been used as a standard technique for triaging most patients suffering from acute cholecystitis. It provides useful diagnosis information for omenta, mesenteries, gut, and peritoneum... etc. with insignificant effect by the bowel gas and fat [6]-[8]. Non-contrast computed tomography (CT) gained important role with high sensitivity (98\%) and specificity (96\% - 98\%) for detection of urinary stones [9].

This study attempted to reveal the efficacy of non-contrast CT during the diagnosis of urinary stones and also to assess the diagnostic performance of the unenhanced and enhanced with contrast phases separately in patients imaged with CT for suspected acute aortic syndromes in patients presenting to the Emergency Department in acute conditions.

\section{Materials and Methods}

After getting the approval of Ethical committee at King Abdulaziz University Hospital, this study retrospectively reviewed electronically available notes for patients admitted to the Emergency Department (ED) over the last year 2013. All patients examined on a multi-slice CT scanner (64 slice Siemens somatom definition dual source), and the contrast administrated using automatic power injector. Different patient preparation and variety Helical CT scanning protocols were selected to understand the nature of diseases causing acute abdomen pain. The imaging protocols selected are based on the manufacturer original settings with the local expertise opinion added, clinical setting and most likely diagnosis. More emphasis on customization of some parameters and factors to patients individually e.g. slice and pitch collimation; implementation of IV, rectal, and oral contrast media; and limited exam or unlimited-focus complete pelvic and abdominal study. Summarized clinical findings, as in routine practice, will be provided. The CT scan was evaluates and records data in a similar way. The Helical CT protocols varied from enhanced using contrast and uenhanced CT of the abdomen and chest for acute aortic syndrome suspects and non-contrasted CT for urinary stone and Helical CT scanning for acute cholecystitis were retrospectively identified as shown bellow (Table 1 and Table 2).

Table 1. Abdomen and pelvic: Urolithiasis. (a) Renal stone scanning protocol; (b) Reconstruction parameters.

(a)

\begin{tabular}{ccc}
\hline Scanning protocol & Acquisition parameters & Comments \\
\hline Scout (topogram) & A-P ${ }^{*}$ & Abdomen/Pelvis \\
Patient position & Prone & \\
Scan range & Top of kidneys to symphysis & Dependent on patient habits \\
Scan direction & Cephalo caudal & \\
Tube voltage (kVp) & 100 or less & \\
Effective mAs & 50 use CARE dose $4 \mathrm{D}$ & On obese patients different collimation applies \\
Gantry rotation time (s) & 0.5 & \\
Slice collimation (mm) & $64 \times 0.6$ & \\
Pitch & 0.9 & \\
Table feed (mm/rotation) & 17.28 & \\
\hline
\end{tabular}

\footnotetext{
${ }^{*}$ Anterior posterior.
} 
(b)

$\begin{array}{cc}\text { Slice width }(\mathrm{mm}) & 2 \\ \text { Axial slice width for 3D/MPR }{ }^{* *}(\mathrm{~mm}) & 2 \\ \text { Recon. increment }(\mathrm{mm}) & 2 \\ \text { Axialrecon. Increment for 3D/MPR }(\mathrm{mm}) & 2 \\ \text { Special views } & \text { Coronal } \\ \text { Recon. Field of view } & \text { Fit to patient }\end{array}$

${ }^{* *}$ Three dimensional/Multi-planer reconstruction.

Table 2. Aorta: Primary diagnosis of suspected abdominal aortic aneurysm or dissection. (a) Contrast protocol; (b) Scanning protocol; (c) Reconstruction parameters.

(a)

\begin{tabular}{|c|c|c|}
\hline \multirow{2}{*}{$\begin{array}{l}\text { Contrast protocol } \\
\text { Enhancement phase }\end{array}$} & \multicolumn{2}{|c|}{ Parameters } \\
\hline & Unenhanced & Arterial \\
\hline IV contrast-iodineconc $(\mathrm{mgl} / \mathrm{mL})$ & & 350 \\
\hline Iodine delivery rate (gl/s) & & 1.9 \\
\hline Volume (mL) & & 120 \\
\hline Flow rate $(\mathrm{mL} / \mathrm{s})$ & & 5 \\
\hline Saline flush-volume (mL) & & 50 \\
\hline Saline flush-flow rate $(\mathrm{mL} / \mathrm{s})$ & & 4.5 \\
\hline
\end{tabular}

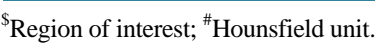

(b)

\begin{tabular}{ccc}
\hline Scanning protocol & & Acquisition parameters \\
\hline Enhancement phase & Unenhanced & Arterial \\
\hline Scout (topogram) & Anterior-Posterior & Anterior-Posterior \\
Patient position & Supine & Supine \\
Scan range & From neck arch to mid femur & From neck arch to mid femur \\
Scan direction & Cephalo caudal & Cephalo caudal \\
Tube voltage (kVp) & 120 & 120 \\
Effective mAs & 200 to 280 & 200 to 280 \\
Gantry rotation time (s) & 0.33 or 0.37 & 0.33 or 0.37 \\
Slice collimation (mm) & $64 \times 0.6$ & $64 \times 0.6$ \\
Pitch & 0.75 or 0.9 (dependent on $\mathrm{mAs}$ ) & 0.75 or 0.9 (dependent on mAs) \\
Table feed (mm/rotation) & 14.4 or 17.28 (dependent on $\mathrm{mAs})$ & 14.4 or 17.28 (dependent on mAs) \\
\hline
\end{tabular}

(c)

\begin{tabular}{|c|c|c|}
\hline Slice width (mm) axial & 5 & 3 \\
\hline Recon. increment (mm) & 5 & 0.75 \\
\hline Special views (mm) & $\mathrm{N} / \mathrm{A}^{+}$ & Coronal/Sagittal, MIP oblique 3D and $\mathrm{VRT}^{\wedge}$ with $5 \mathrm{~mm}$ \\
\hline Recon. Field of view & Fit to patient & Fit to patient \\
\hline
\end{tabular}

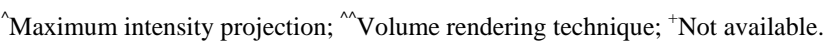




\subsection{General Points Regarding Abdomen and Pelvic Exam}

- If patient is able, scan the patient in the prone position. This is useful for differentiating between an ureterovesical junction (UVJ) stone and a passed stone.

- No patient preparation is required because of this is a non-contrast study ,however , better patient hydration through the ingestion of water before the study can help to eliminate small hyper densities of the renal pyramids that can mimic stones.

- Thin slices allow identification of small stones that may be overlooked with thicker slices.

- The radiation dose, should be kept minimum particularly to the gonads. It is important because many patients who have stone are young and may have repeated stone formation. Therefore, might undergo CT again several times in the future.

Lower dose techniques can reduce the exposure but exposure can still be high if multiple examinations are obtained.

\subsection{General Points Regarding Aorta Exam}

- The patient is prepared, if contrast will be used during an examination, the patient will be asked to fast for several hours before administration (4 - 6 hours), fresh serum creatinine and GFR are required.

- Large coverage area to include subclavian arteries to mid femur to exclude any dissection.

\section{Results}

\subsection{Urolithiasis}

Non-contrasted Helical CT calcified and non-calcified urinary stones were identified, along with the location and size of the stone from kidney to bladder. Secondary signs of obstructive uropathy, including hydronephrosis and ureteral ecstasies were noticed. On non-contrast CT, calcified urinary stones appear as opaque densities within the urinary tract to differentiate calcified and non-calcified urinary stones according to their appearance are not possible. Degree of accuracy in interpreting a non-contrast CT in a case with urinary stone increases in accordance with the severity of urinary obstruction. Examples of such non-contrast Helical CT images of renal stoneare shown in Figures 1-3 which are representing axial, coronal and sagittal views respectively.

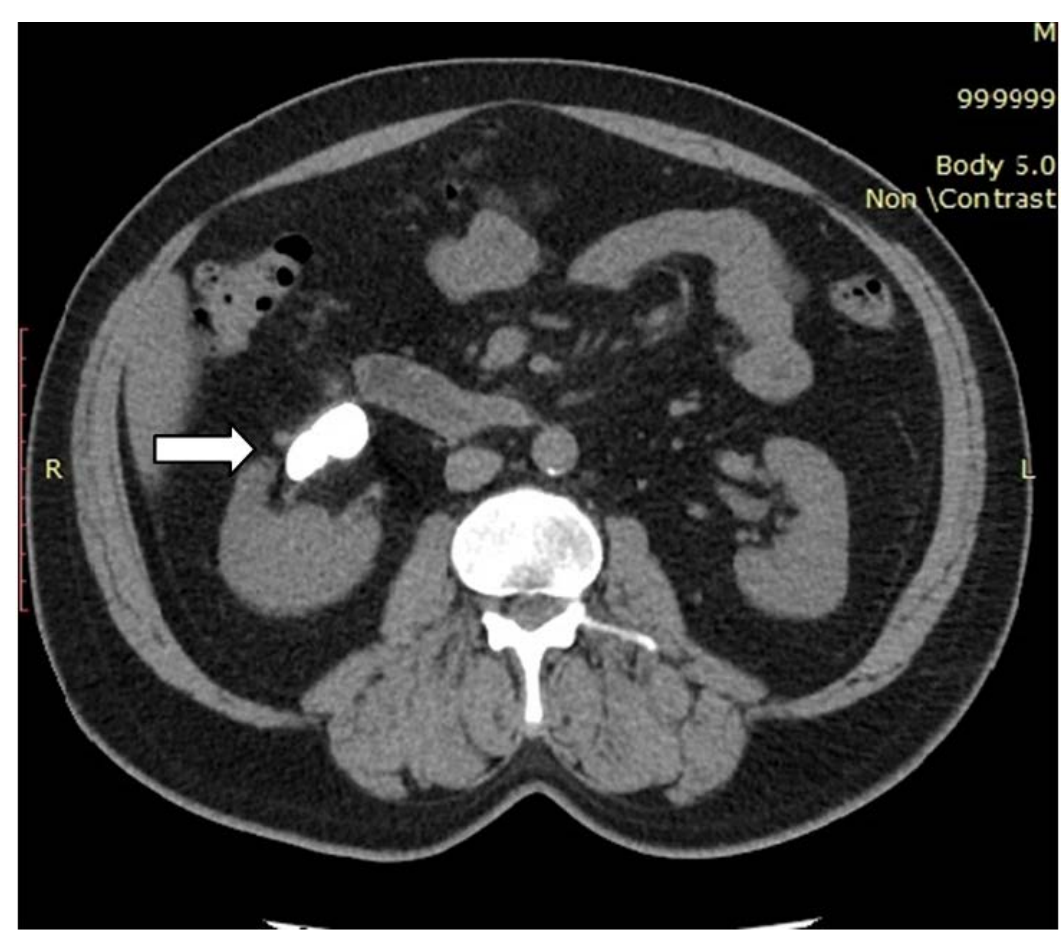

Figure 1. Non-contrast axial CT image showing renal stone. 


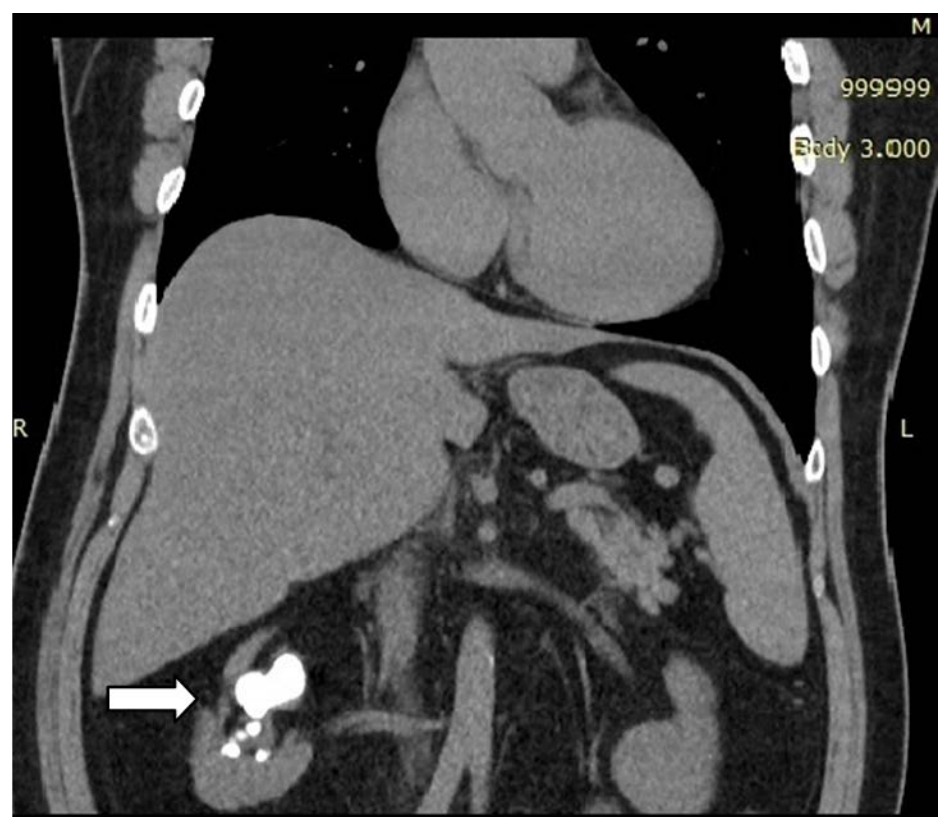

Figure 2. Non-contrast coronal CT image showing renal stone.

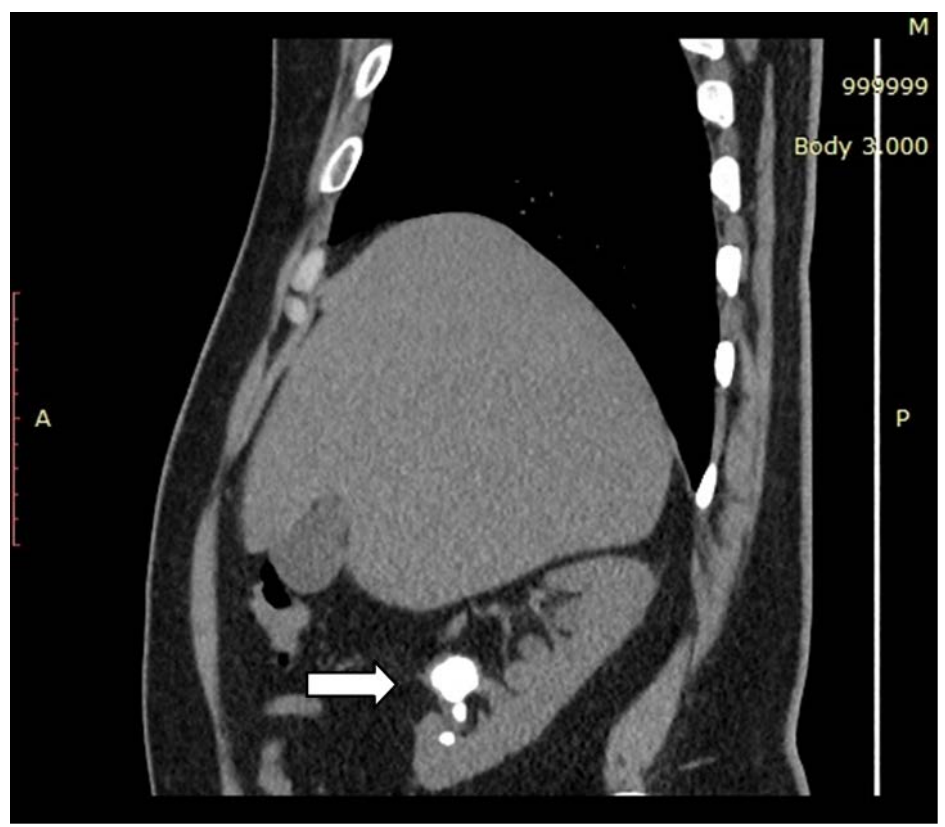

Figure 3. Non-contrast sagittal CT image showing renal stone.

\subsection{Aortic Aneurysm Rupture}

The symptoms of Aortic Aneurysm Rupture (Dissection) can be in the form of abdominal pain, a pulsatile mass, and hypotension. About 30\% of patients do not experience these symptoms and might be misdiagnosed thus reporting renal colic and diverticulitis. Aneurysm rupture was diagnosed in elderly smoking people. Images are obtained to look initially for hyperdense blood related to rupture, the sign for draped aorta; the sign for high-attenuation crescent, attributed to hemorrhage found in mural thrombus or can also be found in the wall of the aneurysm that could be basic sign of aneurysm rupture. Enhancement and perfuse of the atherosclerotic walls of aneurysms is achieved by the vasa vasorum, nonenhancing presented on CT with low density focal areas, and rupture signs containing a retroperitoneal hematoma. Example of these Helical CT images is shown in Figure 4 


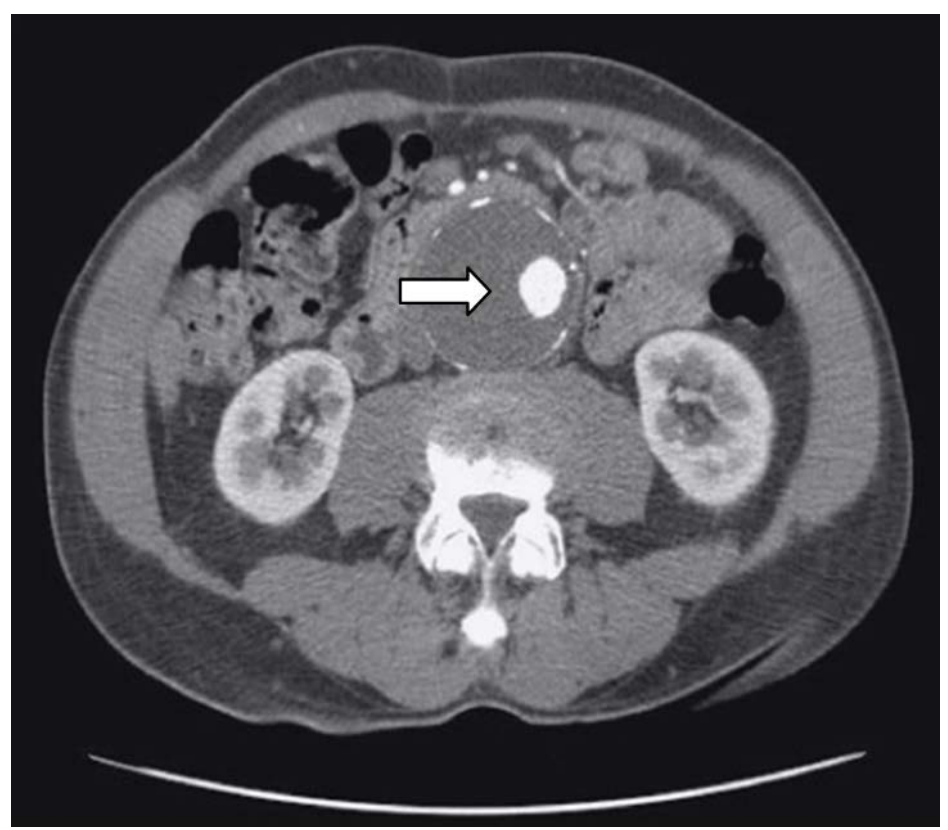

Figure 4. Contrast enhanced axial CT image showing aortic aneurysm.

which is representing contrast enhanced axial CT image showing aortic aneurysm.

\subsection{Acute Cholecystitis}

CT findings of acute cholecystitis were mural thickening and some enhancement to the inflamed wall via transient focal higher attenuation of the liver was developed next to the gallbladder that is inflamed. Secondary signs may be fluids of pericholecystic, pericholecystic fat haziness, and gallbladder bile increased attenuation.

\section{Discussion}

Approximately 30,000 patients with acute abdomen were reviewed in an earlier study [10], the observation revealed that $28 \%$ having appendicitis, $9.7 \%$ showed acute cholecystitis, $4.1 \%$ with small-bowel obstruction, $4 \%$ with acute gynecologic disease, $2.9 \%$ showed acute pancreatitis, $2.9 \%$ with acute renal colic, $2.5 \%$ with perforated peptic ulcer, and $1.5 \%$ with diverticulitis. About $30 \%$ of patients showed no direct cause could be identified.

Recent report [11] indicates that multi-detector row CT urography with multi-planar reformations is helpful in evaluation of the urinary tract. The reported findings of urinary stone in this work were similar to that reported by [12] i.e. with non-contrasted CT, calcified and non-calcified urinary stones was identified, along with the location and size of the stone from kidney to bladder. A secondary sign of obstructive uropathy, including hydronephrosis was noticed. On non-contrast CT, calcified urinary stones appear as opaque densities within the urinary tract. Degree of accuracy in interpreting a non-contrast CT in a case with urinary stone increases in accordance with the severity of urinary obstruction.

Previous studies demonstrated that CT allowed diagnosis and determining the size, composition, and location of stones [13]. This was done by analyzing the correlation between dimensions of stone using CT scan assessment and plain X-ray of the kidneys, bladder and ureter. Other studies [14] [15] to evaluate calculi, it was possible to demonstrate a sensitivity of $97 \%$, specificity of $96 \%$, and accuracy of $97 \%$ in 60 patients. Identification of the number, size, and location of urinary stones and detection of hydronephrosis are easily made with CT.

Ruptured aortic aneurysm symptoms are commonly abdominal pain, hypotension, and a pulsatile mass. Ruptured aneurysm diagnosed in elderly men must be considered if they were smokers due to increased possibility of rupture. It is clear that Helical CT is the chosen modalitiy for those patients possibly having aneurysm dissection together with rupture [5] [16]. An immediate sign of aneurysm rupture; and focal discontinuity of intimal calcification is the increased-attenuation crescent sign, as a result of hemorrhage in mural thrombus or may be 
within the wall of the aneurysm [17].

Oral contrast media is not recommended. Images that are unenhanced are basically obtained to look for the hyperdensed blood related to signs of impending rupture. Infusion of contrast media i.e. $3-4 \mathrm{ml} / \mathrm{sec}$ and thinner collimation of about $5 \mathrm{~mm}$ are used for idealresolution for vascular visualization [18]. In CT images, first signs of rupture show a retroperitoneal hematoma or may be frank extravasation of IV contrast media [19]. For isolated intramural hematoma, enhanced contrast CTA sensitivity reached $100 \%$.

Ultrasound imaging is usually the used method for the diagnosis of acute cholecystitis, but CT examination is best choice in case of uncertain image diagnose. In acute cholecystitis, Helical CT findings are mostly sensitive in mural thickening for more than $3 \mathrm{~mm}$ and inflamed wall enhancement [20].

Because of hepatic artery hyperemia and early venous drainage, transient focal increased attenuation of liver was observed side by side to the inflamed gallbladder [21]. Moreover, Helical CT is capable in providing information for complications of acute cholecystitis e.g. gangrene and perforation.

\section{Conclusion}

According to the findings, non-contrast Helical CT scan performed after ultrasonography is the common diagnostic modality for patients with urinary stones in the emergency department. Enhanced CT with contrast has the highest sensitivity for intramural hematoma. The scanning parameter of the protocols for acute aortic syndrome must undergo customized adjustment to maintain dose from radiation as low as reasonably achievable.

\section{References}

[1] Trott, A.T. and Lucas R.H. (1998) Acute Abdominal Pain. In: Rose, P., Ed., Emergency Medicine, 4th Edition, Mosby, St. Louis, 1888-1903.

[2] Martin, R.F. and Rossi, R.L. (1997) The Acute Abdomen: An Overview and Algorithms. Surgical Clinics of North America, 77, 1227-1243. http://dx.doi.org/10.1016/S0039-6109(05)70615-0

[3] Clouse, W.D., Hallett Jr., J.W, Schaff H.V., et al. (2004) Acute Aortic Dissection: Population-Based Incidence Compared with Degenerative Aortic Aneurysm Rupture. Mayo Clinic Proceedings, 79, 176-180. http://dx.doi.org/10.4065/79.2.176

[4] Olsson, C., Thelin, S., Stahle, E., Ekbom, A. and Granath, F. (2006) Thoracic Aortic Aneurysm and Dissection: Increasing Prevalence and Improved Outcomes Reported in a Nationwide Population-Based Study of More Than 14,000 Cases from 1987 to 2002. Circulation, 114, 2611-2618. http://dx.doi.org/10.1161/CIRCULATIONAHA.106.630400

[5] Siegel, C.L. and Cohan, R.H. (1994) CT of Abdominal Aortic Aneurysms. AJR, 163, 17-29. http://dx.doi.org/10.2214/ajr.163.1.8010207

[6] Siewert, B., Raptopoulos, V., Mueller, M.F., Rosen, M.P. and Steer, M. (1997) Impact of CT on Diagnosis and Management of Acute Abdomen in Patients Initially Treated without Surgery. AJR, 168, 173-178. http://dx.doi.org/10.2214/ajr.168.1.8976942

[7] Malone, A.J. (1999) Unenhanced CT in the Evaluation of the Acute Abdomen. Seminars in Ultrasound, CT and MR, 20, 68-76. http://dx.doi.org/10.1016/S0887-2171(99)90038-0

[8] Mindelzun, R.E. and Jeffrey, R.B. (1999) The Acute Abdomen: Current CT Imaging Techniques. Seminars in Ultrasound, CT and MR, 20, 63-67. http://dx.doi.org/10.1016/S0887-2171(99)90037-9

[9] Wang, J.H., Shen, S.H., Huang, S.S. and Chang, C.Y. (2008) Prospective Comparison of Unenhanced Spiral Computed Tomography and Intravenous Urography in the Evaluation of Acute Renal Colic. Journal of the Chinese Medical Association, 71, 30-36. http://dx.doi.org/10.1016/S1726-4901(08)70069-8

[10] deBombal, F.T. (1991) Introduction. In: deBombal, F.T., Ed., Diagnosis of Acute Abdominal Pain, 2nd Edition, Churchill Livingstone, Edinburgh, 1-10.

[11] Caoili, E.M., Cohan, R.H., Korobkin, M., et al. (2002) Urinary Tract Abnormalities: Initial Experience with Multi-Detector Row CT Urography. Radiology, 222, 353-360. http://dx.doi.org/10.1148/radiol.2222010667

[12] Johnson, E.K., Faerber, G.J., Roberts, W.W., Wolf, J.S., Park, J.M., Bloom, D.A. and Wan, J. (2011) Are Stone Protocol Computed Tomography Scans Mandatory for Children with Suspected Urinary Calculi? Urology, 78, 662-666. http://dx.doi.org/10.1016/j.urology.2011.02.062

[13] Tisdale, B.E., Siemens, D.R., Lysack, J., Nolan, R.L. and Wilson, J.W. (2007) Correlation of CT Scan versus Plain Radiography for Measuring Urinary Stone Dimensions. The Canadian Journal of Urology, 14, 3489-3492.

[14] Liu, W., Esler, S.J., Kenny, B.J., Goh, R.H., Rainbow, A.J. and Stevenson, G.W. (2000) Low-Dose Nonenhanced Helical CT of Renal Colic: Assessment of Ureteric Stone Detection and Measurement of Effective Dose Equivalent. 
Radiology, 215, 51-54. http://dx.doi.org/10.1148/radiology.215.1.r00ap4051

[15] Lin, W.C., Uppot, R.N., Li, C.S., Hahn, P.F. and Sahani, D.V. (2007) Value of Automated Coronal Reformations from 64-Section Multidetector Row Computerized Tomography in the Diagnosis of Urinary Stone Disease. Journal of Urology, 178, 907-911. http://dx.doi.org/10.1016/j.juro.2007.05.042

[16] Adam, D.J., Bradbury, A.W., Stuart, W.P., et al. (1998) The Value of Computed Tomography in the Assessment of Suspected Ruptured Aortic Aneurysm. Journal of Vascular Surgery, 27, 431-437. http://dx.doi.org/10.1016/S0741-5214(98)70317-9

[17] Mehard, W.B., Heiken, J.P. and Sicard, G.A. (1994) High-Attenuating Crescent in Abdominal Aortic Aneurysm Wall at CT: A Sign of Acute or Impending Rupture. Radiology, 192, 359-362. http://dx.doi.org/10.1148/radiology.192.2.8029397

[18] Costello, P. and Gaa, J. (1995) Spiral CT Angiography of Abdominal Aortic Aneurysms. Radiographics, 5, $397-406$. http://dx.doi.org/10.1148/radiographics.15.2.7761643

[19] Mackiewicz, Z., Molski, S., Szpinda, M., Jundzill, W. and Stankiewicz, W. (1998) Retroperitoneal Rupture of Abdominal Aortic Aneurysms. Journal des Maladies Vasculaires, 23, 368-370.

[20] Fidler, J., Paulson, E.K. and Layfield, L. (1996) CT Evaluation of Acute Cholecystitis: Findings and Usefulness in Diagnosis. American Journal of Roentgenology, 166, 1085-1088. http://dx.doi.org/10.2214/ajr.166.5.8615248

[21] Yamashita, K., Jin, M.J., Hirose, Y., et al. (1995) CT Findings of Transient Focal Increased Attenuation of the Liver Adjacent to the Gallbladder in Acute Cholecystitis. American Journal of Roentgenology, 164, 343-346. http://dx.doi.org/10.2214/ajr.164.2.7839966 
Scientific Research Publishing (SCIRP) is one of the largest Open Access journal publishers. It is currently publishing more than 200 open access, online, peer-reviewed journals covering a wide range of academic disciplines. SCIRP serves the worldwide academic communities and contributes to the progress and application of science with its publication.

Other selected journals from SCIRP are listed as below. Submit your manuscript to us via either submit@scirp.org or Online Submission Portal.
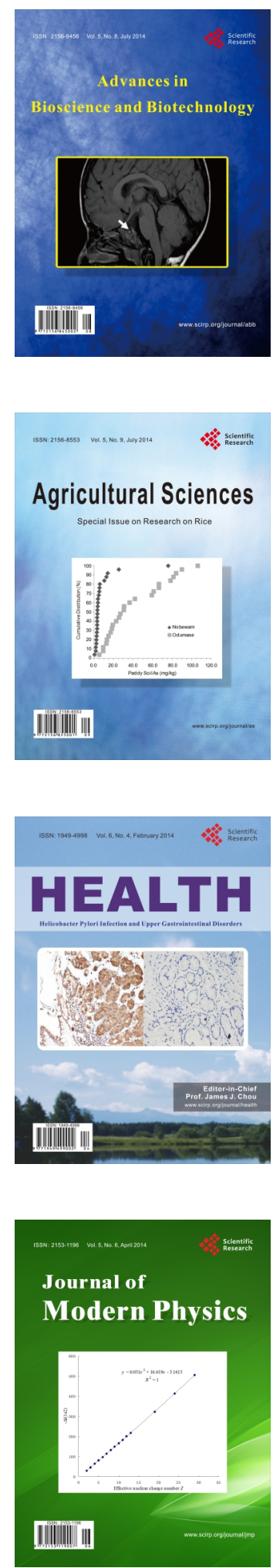
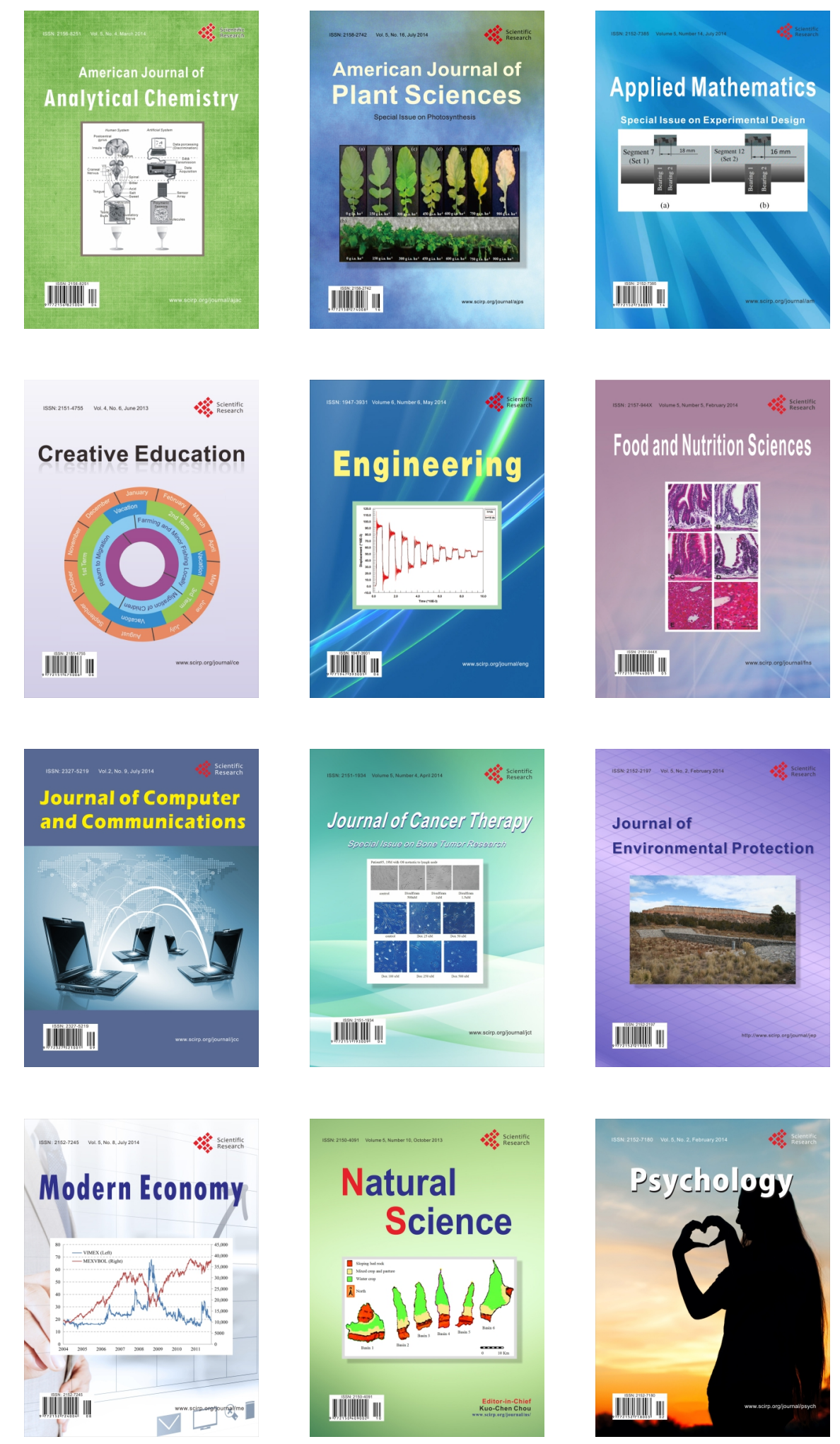